\section{Palobra}

acceso $\partial$ abierto

Cómo citar: Maldonado, J. y Mosquera, J. (2020). Teatro foro y pedagogía activa como estrategia para la gestión de conflictos y la transformación social. Palobra, 20(2), 134149. 10.32997/2346-2884-vol.20-num.2-2020$\underline{3312}$

Recibido: 6 de mayo de 2020 .

Aprobado: 12 de agosto de 2020 .

Autor de correspondencia:

Jaqueline Maldonado Avendaño

jarfem@unipamplona.edu.co

Editora: Rosario Blanco Bello. Universidad de Cartagena-Colombia.

Tipología IBN Publindex: Artículo de Reflexión.

\title{
Teatro foro y pedagogía activa como estrategia para la gestión de conflictos y la transformación social $^{1}$
}

\author{
Forum theater and active pedagogy as a strategy for conflict \\ management and social transformation
}

Jaqueline Maldonado Avendaño

Universidad de Pamplona, Colombia, jarfem@unipamplona.edu.co

Jemay Mosquera Téllez

Universidad de Pamplona, Colombia, jemay.mosquera@unipamplona.edu.co

\section{RESUMEN}

El documento se fundamenta en los postulados de la pedagogía activa y los métodos del teatro foro como estrategia alternativa, que puede aportar a la transformación sociocultural y la gestión pacífica de conflictos en jóvenes universitarios. Se asume la metodología cualitativa, método fenomenológico, con alcance exploratorio, y parte de aspectos teóricos y conceptuales sobre la pedagogía activa y liberadora, el teatro foro, el conflicto social y la gestión de conflictos, como base para proponer y desarrollar una secuencia procedimental asociada a la aplicación del teatro foro como herramienta para la exploración de conflictos presentes en los contextos sociales de la población objetivo. Finalmente, los resultados obtenidos están relacionados con la formulación, implementación y validación de una estrategia artística y pedagógica basada en lineamientos teórico- prácticos de la pedagogía activa, las técnicas, juegos y ejercicios asociados al teatro foro, que puede aportar a la transformación de las experiencias vividas y observadas de los estudiantes.

Palabras clave: gestión de conflictos; pedagogía activa; teatro foro; transformación sociocultural.

\begin{abstract}
The document is based on the postulates of active pedagogy and the methods of forum theater as an alternative strategy, which can contribute to the sociocultural transformation and the peaceful management of conflicts in young university students. The qualitative methodology, phenomenological method, with an exploratory scope, and part of theoretical and conceptual aspects about active and liberating pedagogy, forum theater, social conflict and conflict management are assumed; as a basis for proposing and developing a procedural sequence associated with the application of forum theater as a tool for exploring conflicts present in the social contexts of the target population. Finally, the results obtained are related to

\footnotetext{
${ }^{1}$ Este artículo es producto del proyecto de investigación titulado "El teatro foro y la pedagogía activa como estrategia didáctica de transformación sociocultural. Un aporte a la gestión de conflictos y la construcción de cultura de paz" y fue financiado con recursos propios.
}

Copyright: (1) 2020. Maldonado, J. y Mosquera, J. Este es un artículo de acceso abierto, distribuido bajo los términos nc-sa/4.0/ la cual permite el uso sin restricciones, distribución y reproducción en cualquier medio, siempre y cuando que el original, el autor y la fuente sean acreditados.

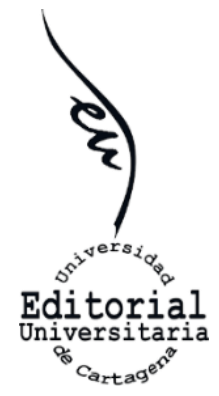


the formulation, implementation and validation of an artistic and pedagogical strategy based on theoretical-practical guidelines of active pedagogy, techniques, games and exercises associated with forum theater, which can contribute to the transformation of the lived and observed experiences of students.

Keywords: conflict management; active pedagogy; forum theater; sociocultural transformation.

\section{INTRODUCCIÓN}

A pesar de la existencia de organismos institucionales y bases teóricas orientados a resolver los conflictos de los estudiantes universitarios, se evidencia que esos no son conscientes del origen o tipología de sus conflictos personales o sociales, mientras que las estrategias que se implementan para su gestión no siempre logran su objetivo. Por lo tanto, el presente estudio fenomenológico busca aportar a la disminución de estas inconsistencias y corresponde a un trabajo que se viene desarrollando desde el año 2015, con el propósito de explorar los conflictos mediante la aplicación de una estrategia fundamentada en los postulados de la pedagogía activa de Paulo Freire (1996, 1984, 1086, 2009) y las técnicas de teatro foro concebidas por Augusto Boal (2001, 2004 y 2012), las cuales están representadas en técnicas, ejercicios físicos y juegos teatrales que fueron aplicadas en jóvenes universitarios para buscar un reconocimiento personal, como ser social; asimismo, convertir su rol de espectador pasivo a sujeto transformador de la acción dramática, preparándolo para que pueda transponer a la vida real acciones que ha ensayado en la práctica teatral. En ese sentido, el trabajo se orienta a establecer la forma de cómo se puede realizar una transformación social a través del teatro foro; por tal razón, se debe partir desde la perspectiva del teatro del oprimido planteado por Augusto Boal, como un espacio social que permite visibilizar las opresiones de las personas y producir liberación para que se transformen comunitariamente, se reedifiquen como comunidad sin ser limitado al teatro en sí mismo (Boal, 2015). De igual forma, se basa en el teatro dialéctico de Bertolt Brecht que plantea una concienciación y se contrapone a la clásica griega propuesta por Aristóteles sobre el teatro como una catarsis. Por consiguiente, en el marco de la pedagogía del oprimido se busca tomar conciencia de las relaciones de opresión, en cuanto al rol del opresor y el oprimido, entendido a partir de la teoría de la violencia estructural enunciada por Galtung, cuyas raíces pueden ser económicas, políticas, sociales y culturales; así como la violencia directa, la cual también permite identificar relaciones de dominación y sometimiento, sin fragmentar la realidad o aislar los problemas. Además, busca resaltar el papel del teatro foro como una disyuntiva no violenta que, frente a las violencias, promueve el diálogo y la exploración de alternativas para afrontar situaciones conflictivas, por tanto, aunque se ensaye la violencia, el teatro siempre devela su inoperancia, incitando y desafiando la capacidad creativa para transformarla 
en un ensayo de resistencia civil activa, como instrumento de trabajo terapéutico para la gestión pacífica de los conflictos.

Cabe señalar, que el objetivo general se orienta en implementar una estrategia fundamentada en los postulados de la pedagogía activa y las técnicas de teatro foro que aporte a la gestión de conflictos y la transformación sociocultural; así como, específicamente, se propuso identificar los aspectos teóricos y conceptuales que tienen mayor y más estrecha relación de interdependencia entre el teatro foro, la pedagogía activa, el conflicto, la gestión de conflictos y la transformación social; por otra parte, se propuso desarrollar una secuencia metodológica basada en el teatro foro y aplicar la propuesta en jóvenes universitarios para la exploración de conflictos presentes, así como la interpretación de los mismos, lo que permitió la definición del problema de investigación y la reflexión sobre este, también la identificación de categorías y temas esenciales del teatro foro.

Por lo tanto, se asume la estructura metodológica cualitativa, de alcance exploratorio y método fenomenológico, se llevó a cabo mediante el análisis y triangulación de las técnicas de recolección de información, como un proceso dirigido a la comprensión de lo que ocurre, mientras se involucra la conducta humana desde una estrategia artística. De esta manera, a través del teatro y las técnicas dramáticas, se logra comprender las causas de los conflictos sociales e interpersonales, liberar mediante el juego, facilitar la búsqueda de alternativas para transformar y gestionar conflictos a través de la poética del oprimido, y fortalecer la autoestima, la expresión teatral y la creatividad. Además, el trabajo posibilitó la generación de espacios de atención para avivar los sentidos, la exploración y la sensibilidad hacia sí mismo y el otro, hacia el entorno social y el medio natural.

Como resultado, se comprueba el cumplimiento de los objetivos propuestos, también se establece que la propuesta conlleva a un enfoque de praxis pedagógico y artístico, centrándose en las posibilidades humanas de creatividad y libertad que orientan al descubrimiento y aplicación de soluciones liberadoras, por medio de la interacción y la transformación personal, concienciando, tanto de la realidad sociocultural como de la capacidad de transformar esa realidad.

\section{Metodología}

El estudio corresponde al campo de la investigación cualitativa y busca hacer una lectura de la realidad de manera holística acudiendo a observaciones naturalistas y modelos intensivos y comprensivos, de manera constructivista y dialógica, con carácter inductivo y particularista, cuya esencia primordial es el interés de captar la percepción del sujeto de su propio contexto. Por consiguiente, la construcción de conocimiento surge de la indagación guiada por un diseño emergente estructurado a partir de hallazgos sucesivos 
obtenidos en el transcurso de la investigación y de la validación de las conclusiones obtenidas a través de diálogo, interacción, reflexión, construcción de sentido compartido y sistematización. Cabe señalar que la aplicación de la fenomenología hermenéutica, consistente en la interpretación de experiencias humanas sin seguir reglas específicas, que permite la definición del problema de investigación, la reflexión sobre el mismo, la identificación de categorías y temas fundamentales del teatro foro que constituye la naturaleza de la experiencia y su interpretación mediante diferentes significados aportados por los participantes (Creswell, 1998).

En el marco de lo enunciado y de la pregunta problematizadora: ¿De qué manera se puede inferir situaciones cotidianas de conflicto como transformación social, que permitan la gestión de los mismos, y potenciar el aprendizaje en jóvenes universitarios desde procesos artísticos como el teatro foro? En el contexto de la propuesta metodológica y para efecto de su implementación, las herramientas de recolección y análisis de información permitieron describir e interpretar los fenómenos desde el punto de vista de cada participante, y desde la perspectiva construida colectivamente, teniendo en cuenta el contexto de las experiencias en términos de su temporalidad, espacialidad, corporalidad y contexto relacional. La población objetivo corresponde a una escuela de formación teatral universitaria y la muestra se realizó con ocho estudiantes: cuatro hombres y cuatro mujeres, de forma voluntaria y con el consentimiento informado de cada participante y el aval del comité de ética e impacto ambiental institucional. En el marco de los objetivos propuestos, se plantearon cinco categorías: teatro foro, pedagogías activas y liberadoras, conflicto, gestión de conflictos y transformación sociocultural.

\section{Fundamentación teórica}

La fundamentación teórica y conceptual está relacionada con dos categorías conceptuales: gestión de conflictos; teatro foro y pedagogía activa, las cuales se enuncian a continuación.

\subsection{Gestión de conflictos}

Estas líneas intentan indagar inicialmente en el análisis del conflicto, para seguidamente abordar la gestión de conflictos; resaltando que la propiedad del conflicto es inherente a la naturaleza humana y su incidencia en nuestras relaciones.

Entonces, el conflicto es un proceso natural de la sociedad y un fenómeno necesario para la vida humana, pudiendo ser un factor positivo para el cambio y el crecimiento personal e interpersonal o un factor negativo de destrucción, según la forma de regularlo. El conflicto no es ni bueno ni malo, simplemente existe. Es como una fuerza natural que, controlada y en su justa y equilibrada 
fuerza, puede desarrollar a la naturaleza, producir energía y estimular la vida $\mathrm{y}$, por otra parte, cuando se presenta en forma descontrolada, puede alterar los ciclos naturales, destruir e impedir el crecimiento de la vida (Muñoz, 2003).

Por lo anterior, para Johan Galtung desde una perspectiva de causas estructurales de los conflictos que parten del individuo y de sus necesidades sociales, como proceso que se orienta primordialmente a eliminar tres formas de violencia: directa (agresión física directa), estructural (proveniente de las estructuras sociales, políticas y económicas opresivas que impiden el desarrollo de las personas) y cultural (asociada a la imposición de valores o pautas culturales que niegan la diversidad cultural y legitimando el uso de la fuerza para resolver los conflictos), de tal forma que paz no equivale únicamente a ausencia de conflicto o guerra, sino a la ausencia de violencia en todas sus formas (Galtung, 1976), aspecto que fue ratificado por las Naciones Unidas con la promulgación de una cultura de paz que sustituye de las tendencias de guerra y de violencia dominante (Unesco, 1998; Robles \& Ortiz, 1998).

Dentro de este contexto, según Galtung esto se logra mediante el proceso de concientización, siguiendo la metodología de Paulo Freire (1921-1997). La propuesta del pedagogo brasileño se caracteriza más que por los métodos empleados, cuanto por el estrecho vínculo que establece entre el aprendizaje linguístico y la toma de conciencia frente a la realidad. Su programa es un continuo llamamiento educativo para que los pobres y explotados a través de la toma de conciencia de su realidad busquen sus propios caminos de liberación, presentes en su condición histórica. Para el autor contar con una imagen consciente y cabal del conflicto, con todos sus aspectos profundos, sus condicionantes históricos, será una condición sin la cual, no para la transformación del mismo. El proceso de concientización evitará la cosificación de los actores haciendo de ellos protagonistas del proceso, es decir, "un actor consciente será capaz de dirigir esa transformación incluyendo la propia" (Galtung, 2003, p. 112).

Por consiguiente, "el conflicto puede enfocarse básicamente como una de las fuerzas motivadoras de nuestra existencia, como una causa, un concomitante y una consecuencia del cambio" (Galtung, 1981, p. 11), y puede ser considerado como un instrumento esencial para la transformación de las estructuras educativas y las estructuras sociales e institucionales que enmarcan la actuación de los protagonistas del conflicto (Carr y Kemmis, 1986).

En el marco de la gestión de conflictos, la evolución de los estudios de la paz (Muñoz y Molina, 2009) y de la educación para construir cultura de paz (Tuvilla, 2004; Hernández Arteaga, Luna Hernández y Cadena Chala, 2017), así como de la transformación pacífica de conflictos (Paris y Martínez, 2009), se ha pasado por diversas etapas: la interpretación de la resolución, la gestión 
y la transformación pacífica de los conflictos. La resolución de conflictos surge a mediados del siglo XX y hace énfasis en la acepción negativa de los conflictos y sus consecuencias destructivas (De Armas y Carreiro, 2013; Vinyamata, 2015); la gestión de conflictos se utiliza a partir de los años setenta del siglo pasado y tiene en cuenta las consecuencias destructivas de los conflictos y su relación con la violencia; y la transformación pacífica de los conflictos, utilizada desde la década de los noventa y hasta la actualidad, surge de la carencia de éxito de la resolución y la gestión de conflictos y concuerda con los valores de la cultura de paz al interpretar los conflictos como situaciones de cambio en las que lo más importante es abordar las tensiones que los causaron, con el fin de transformarlas en nuevos objetivos encaminados hacia el restablecimiento de las relaciones en el futuro (Lederach, 2000 y 2003).

De esta manera, la transformación pacífica de los conflictos se da como una alternativa de las regulaciones de los conflictos en la que predomina el valor de los medios pacíficos para afrontarlos frente al uso de la violencia y la aplicación de principios básicos, tales como la no violencia, la creatividad y la empatía, lo que permite asumir el conflicto como una oportunidad para el crecimiento y desarrollo de las partes involucradas (Galtung, 2004) a través de aspectos clave de la moral humana como la revalorización propia y el reconocimiento del otro (Robles \& Ortiz Granja, 2017).

\subsection{La pedagogía activa y el teatro foro}

Desde el campo de la pedagogía, se tienen en cuenta los aprendizajes del conocimiento que desarrolla cada individuo a lo largo de su vida: aprender a conocer, aprender a hacer, aprender a vivir juntos, y aprender a ser (Delors, 1996) y se propone abordar dos enfoques, el de pedagogía activa, fundamentado en los postulados de Piaget relacionados con la inteligencia humana y la forma como esta se estructura para articular el conocimiento y el conflicto en aras de lograr un equilibrio cognitivo que permita mejorar la razón y la convivencia social; y el de la pedagogía teatral, a partir de las concepciones sobre el teatro dialéctico de Bertolt Brecht, las nociones teóricas de Freire y la praxis de Augusto Boal, las cuales buscan la reflexión y el cambio en las relaciones del sujeto con la naturaleza y la sociedad mediante diversas técnicas de enseñanza asociadas a modelos de ruptura, cambio y transformación.

Según la pedagogía activa y de acuerdo con los aportes de Paulo Freire, el interés educativo se centra en las posibilidades humanas de creatividad y libertad en medio de estructuras político-económicas y culturales opresivas, se orienta al descubrimiento y aplicación de soluciones liberadoras por medio de la interacción y la transformación social a través de la concienciación, tanto de la realidad sociocultural como de la capacidad de transformar esa realidad (Freire, 1971). 
Por su lado, la pedagogía teatral presenta vertientes, tales como la pedagogía del oprimido y la pedagogía liberadora. La primera, evade los modelos de adaptación o de transición social y está comprometida con la integración del estudiante con su realidad sociocultural y la pérdida del miedo a la libertad (Freire, 1986); con la disposición de establecer en el estudiante un proceso de recreación, de búsqueda, de independencia y de solidaridad (Freire, 1996), por medio del análisis de las causas que pueden llegar a oprimir a una persona, y de cómo hacer para transformar esta situación; mientras que la pedagogía liberadora reconoce al estudiante como ser inconcluso o imperfecto que debe realizarse dentro de una situación histórica que debe ser transformada a través de la praxis y la acción de sujetos que son simultáneamente profesores y estudiantes, y su objetivo principal es la libertad políticosocial del estudiante mediante la lectura (Freire, 1984) y la exaltación del sentido crítico a través del diálogo (Freire, 2009).

En concomitancia con lo anterior, se propone el teatro del oprimido como un escenario que facilita el reconocimiento de la naturaleza y origen de las opresiones más profundas para poderlas transformar y tiene por objetivo utilizar el teatro y la dramatización como un instrumento eficaz para la comprensión y la búsqueda de alternativas a problemas sociales, interpersonales e individuales, así como alterar las convenciones que dominan el mundo de la representación, transformando al espectador en protagonista de la acción dramática para que pueda preparar acciones reales que le conduzcan a la propia liberación (Boal, 2015). Lo anterior, ha dado como resultado el Teatro Legislativo, el Teatro Invisible, el Teatro Imagen y el Teatro Fórum, o Foro, y la práctica teatral en su vertiente terapéutica conocida como El arco iris del deseo.

Puntualmente, el teatro foro tiene influencia del teatro dialectico y de concienciación de Brecht, se basa en los postulados la Pedagogía Activa y del Oprimido de Freire y es uno de los métodos del Teatro del Oprimido de Augusto Boal. El teatro foro es una herramienta que permite construir la participación del público como espectador-participante y su objetivo fundamental es plantear una problemática de discusión colectiva, en donde todos los participantes pueden incluir su voz, mientras se construyen nuevas formas de solución y comprensión del caso; parte de la selección de una situación o situaciones relevantes para la comunidad con la que se va a trabajar, y continúa con el montaje de una escena alrededor de la temática escogida y, una vez terminada la representación, se procede a la recolección de la idea principal y la indagación al público sobre si está de acuerdo con la solución propuesta a la problemática planteada (Boal, 2001).

El teatro foro no solamente está basado en aspectos relacionados con el teatro, sino también con diferentes formas de arte y, reúne un conjunto de ejercicios, juegos y técnicas teatrales que pretenden la des-mecanización física e intelectual de sus practicantes y la democratización del teatro. Su objetivo es 
utilizar el teatro y las técnicas dramáticas como un instrumento eficaz para la comprensión y búsqueda de alternativas a problemas sociales e interpersonales, por medio de la estimulación a los participantes no-actores a expresar sus vivencias de situaciones cotidianas de opresión a través del teatro. Desde sus implicaciones pedagógicas, sociales, culturales, políticas y terapéuticas, el teatro foro propone transformar al espectador, ser pasivo, en espect-actor, en protagonista de la acción dramática, sujeto creador, estimulándolo a reflexionar sobre su pasado, modificar la realidad en el presente y crear su futuro (Boal, 1989, citado por Vieites García, 2015). El teatro foro busca que los participantes reflexionen sobre las relaciones de poder, mediante la exploración y representación de historias entre opresores y oprimidos, en las que el público asiste y participa de la pieza. Estas propuestas teatrales son construidas en equipo, a partir de hechos reales y de problemas típicos de una comunidad, tales como la discriminación, los prejuicios, la violencia, la intolerancia y otros.

\section{Resultados}

\subsection{Propuesta de teatro foro como estrategia de transformación social}

La propuesta de teatro foro como estrategia de transformación social responde al objetivo central del trabajo, parte de la fundamentación teórica y conceptual relacionada con dos categorías conceptuales: gestión de conflictos; teatro foro y pedagogía activa.

De esta manera, la primera categoría, gestión de conflictos, se interpreta con la transformación pacífica de los mismos, donde la estrategia pedagógica y artística se da como una alternativa de las regulaciones de los conflictos, en la que predomina el valor del teatro foro, para afrontarlos frente al uso de violencias o problemáticas personales y sociales, entonces la aplicación de principios básicos, tales como la no violencia, la creatividad y la empatía, lo que permite asumir el conflicto como una oportunidad para el crecimiento y desarrollo de las partes involucradas.

Seguidamente, en la segunda categoría la pedagogía activa y el teatro foro, se establece que el teatro foro se basa en postulados de la pedagogía activa y del oprimido de Freire y es uno de los métodos del teatro del oprimido de Augusto Boal. Igualmente, se interpreta como una herramienta que permite construir la participación de los jóvenes universitarios, cumpliendo con su propósito primordial que es plantear problemáticas de discusión colectiva, en donde todos los participantes construyen nuevas formas de solución y comprensión de realidades tanto personales como sociales; así, se establece una situación o situaciones relevantes con la que se va a trabajar. Por tanto, se demuestra que el teatro foro y las técnicas dramáticas son un instrumento eficaz para la comprensión y búsqueda de alternativas a problemas sociales e interpersonales, por medio de la estimulación a los participantes a expresar 
sus vivencias de situaciones cotidianas de opresión a través del teatro. Desde sus alcances pedagógicos, sociales, culturales, políticos y terapéuticos, el teatro foro transforma al sujeto pasivo en sujeto creador, estimula a reflexionar sobre su pasado, modificar la realidad en el presente y crear su futuro.

Igualmente, la propuesta de teatro foro como estrategia de transformación social se estructura en dos fases metodológicas: Formulación y Aplicación. La primera fase, está dirigida a la definición de una secuencia metodológica para la aplicación del teatro foro como estrategia artística y pedagógica de transformación sociocultural, la cual comprende a su vez, la exploración de conflictos presentes en la vivencia de estudiantes universitarios y la interpretación de las situaciones de conflictos emergentes en el teatro foro de acuerdo con las categorías propuestas, de la siguiente manera: i) aprestamiento para la definición de la población objetivo y las herramientas de recolección de información, ii) aplicación de las herramientas, iii) evaluación del proceso y iv) socialización de los resultados obtenidos; por su lado, la segunda fase propuesta, corresponde específicamente al desarrollo del trabajo con el grupo focal, la realización de entrevistas, la organización y ejecución de los laboratorios de creación basados en el teatro foro y el diligenciamiento del diario de campo; la sistematización y análisis de los resultados parciales y la triangulación de las técnicas de recolección de información; la identificación de las conclusiones del trabajo y la socialización y publicación de resultados.

A continuación, articula simultáneamente la formulación de la propuesta enunciada y un ejemplo de su aplicación, con lo cual se busca demostrar la efectividad del teatro foro como estrategia pedagógica de transformación social. Luego del aprestamiento obtenido por medio de los procesos previos enunciados en la introducción, se definió una serie de técnicas de recolección y análisis de información: grupo focal, entrevistas semiestructuradas, observación no participante a través de diario de campo y laboratorios de creación.

En el trabajo con el grupo focal se abordaron conceptos como gestión de conflictos, pedagogía activa y teatro foro; se plantearon las categorías de forma general y se compartieron y analizaron experiencias, realidades, emociones, sucesos y temas asociados a los conflictos personales de opresión, voluntariamente tratados desde sus propias percepciones. El interés del proceso se centró en la forma como los estudiantes construyen una perspectiva de cada problema planteado, a través de la interacción. Por consiguiente, producto del trabajo con el grupo focal, los estudiantes formaron un esquema o perspectiva del problema que se planteó, a través de las interacciones desarrolladas de manera colectiva, mientras que la moderación de las sesiones facilitó el logro de los resultados esperados en cuanto al origen y manejo de las emociones, al tratamiento del significado del 
conflicto en el lenguaje particular de los estudiantes, con un alto nivel de profundización, y al estímulo de la participación e interacción de todos respetando la libertad de expresión y los tiempos de intervención.

Igualmente, las entrevistas semiestructuradas fueron enfocadas a la identificación de la percepción de los estudiantes en torno a las dos categorías previamente definidas, lo cual se logró a través de definiciones introductorias y preguntas orientadoras, tanto en aspectos generales sobre el conflicto, la pedagogía activa y el teatro foro, como en aspectos puntuales relacionados con la identificación de las situaciones de conflicto emergentes en el teatro foro y dirigidos a la reconstrucción de la historia que generó dichas situaciones de conflicto, las contribuciones a su transformación personal, la experiencia en el teatro foro y su aporte a la gestión de conflictos. De igual modo, se indagó sobre las necesidades personales en cuanto a sus propuestas artísticas como posibles alternativas de solución y aplicación en situaciones cotidianas y reales de conflicto, con el propósito de mejorar el potencial de aprendizaje y sus relaciones con una proyección social hacia la gestión de conflictos.

Ahora bien, las entrevistas fueron articuladas con las formas de estructuración de los procesos participativos en cuanto a toma de conocimiento, de consciencia y de posición por parte de la población objetivo, y se aplicaron a los ocho participantes de la escuela de formación teatral del centro de bienestar universitario. En este contexto, las categorías: teatro y pedagogía se enfocan en el conocimiento, es decir, en la identificación de la percepción de la población objetivo con relación a los conceptos centrales del trabajo; mientras que la categoría: gestión de conflictos, se relaciona con la toma de posición frente al conocimiento aplicado, ya que pretenden establecer la incidencia y el impacto que pueden generar los aspectos clave del proyecto en la población objetivo. En relación con los conflictos personales, tres estudiantes mencionaron aspectos asociados con mal carácter, dos se refirieron a su falta de tolerancia, y los demás, desde una percepción individual, manifestaron conflictos de inseguridad, soledad, impaciencia, exagerada disposición a colaborar y baja responsabilidad con las obligaciones personales. Esta identificación les permitió abordar de manera teatral sus propuestas personales y colectivas, y ensayar posibles soluciones o manejos para enfrentar cada conflicto.

Por otra parte, la observación no participante se orientó en identificar fenómenos relacionados con la multiplicidad de comportamientos generados a partir de relaciones e intervenciones en problemáticas sociales, así como su convergencia con la propuesta de pedagogía teatral. De igual modo, su buscó obtener datos directos sobre los asuntos velados y signos no verbales que se pudieran percibir y captar en las manifestaciones de los estudiantes. La observación no participante se desarrolló por medio del diario de campo y los laboratorios de creación. Así, el diario de campo abarcó simultáneamente dos 
aspectos: las observaciones y las deducciones. El primer aspecto, se efectuó a través de la técnica de observación directa al momento de realizar el diagnóstico de carácter cualitativo y permitió percibir actuaciones individuales y colectivas, que se conciben de las interacciones e intervenciones que realizaron los estudiantes, las cuales se plasmaron en el Diario Campo. En este sentido, se registraron los fenómenos observados de las experiencias vividas con el grupo de jóvenes universitarios. En primer lugar, la relación directa con el grupo permitió indagar acerca de cómo piensan, sienten, viven y cuáles son sus conflictos personales; y cuál es su percepción de su contexto social, específicamente sobre los comportamientos generados, producto de sus relaciones e intervenciones en torno a problemáticas identificadas con ayuda de la estrategia de la pedagogía teatral. De igual modo, se consignaron asuntos velados y signos no verbales que se descubrieron y captaron en las interacciones e intervenciones. Se confirmó que el grupo está conformado por personas con características propias, producto de sus identidades culturales, realidades, contextos e intereses particulares que los llevaron a participar en el teatro. En particular, las relaciones desarrolladas se enmarcan en la importancia de los sentimientos y el bienestar colectivo; en el incremento de amabilidad, respeto, confianza, seriedad e, interés por lo que piensa y realiza el compañero y en el apoyo mutuo para plantear situaciones reales asociadas a cada uno de los integrantes. Lo anterior, genera un espacio propicio para el aprendizaje, ya que se establecen diferencias en las actuaciones y se definen las formas para aprovechar tales contrastes, se desarrollan intervenciones que ayudan a transformar comportamientos sin ningún tipo de presiones, se participa e interactúa buscando apoyar sin cuestionar o juzgar, se escucha y analiza cada planteamiento o propuesta con el ánimo de aportar al grupo y a sus integrantes. Como resultado, se observa una mejor comunicación del grupo basada en el diálogo, la concertación y el fortalecimiento de las relaciones sociales de cada integrante. Además, se obtuvo una serie de logros sustanciales asociados a la liberación personal, reflexión e interpretación de conflictos propios o cercanos, búsqueda de una gestión pacífica de dichos conflictos, interacción con el público al hacerlo partícipe de la acción dramática, y apropiación del teatro como herramienta que ayuda a los demás en la transformación de conflictos.

Asimismo, los laboratorios de creación, fueron diseñados a través de ejercicios de calentamiento, técnicas y juegos de teatro foro que permitieron la exploración corporal, las interacciones desarrolladas a través de dichas actividades teatrales, el planteamiento de situaciones de conflictos generados en el marco de sus relaciones sociales y la formulación de propuestas escénicas sobre posibles soluciones a esas problemáticas sociales, dando relevancia a sus propias experiencias. En consecuencia, los laboratorios de creación, fueron estructurados en tres etapas dirigidas al conocimiento del cuerpo, la expresividad corporal y la apropiación del teatro como lenguaje, fueron desarrolladas en 13 talleres. Al respecto, los participantes lograron 
desinhibición en la exploración anatómica y, consecuentemente, una mejor expresión corporal, perdieron el miedo y consiguieron avanzar en la expresión verbal y su comunicación en público; también se evidenció progreso y satisfacción al interactuar con las demás personas por medio de su actuación y por la libertad de expresar lo que pensaban sin prejuicios o presiones sociales. A través de los talleres, los participantes identificaron sus conflictos personales y diferentes problemáticas sociales, compartieron sus vivencias y realidades, y propusieron formas creativas de visibilizar conflictos colectivos relacionados con la violencia estructural y la violencia cultural, lo que permitió la interpretación de estas realidades y la propuesta de posibles soluciones aplicadas en los contextos particulares que originaron los conflictos.

Por tal razón, la mayoría de los estudiantes considera que son pasivos frente a conflictos sociales, pero están dispuestos a resolverlos dialógicamente, mientras que otros se perciben siempre activos y dispuestos a dialogar desde una posición proactiva. Respecto a los aportes del teatro foro a la gestión de conflictos, y a su transformación sociocultural, todos los estudiantes consideran que han cultivado empatía; la mayoría de las percepciones coinciden en aspectos asociados a la posibilidad que brinda el teatro para entender, visibilizar y representar la opresión y los conflictos sociales desde una posición proactiva; gestionar los conflictos personales y colectivos y fortalecer la tolerancia, el compañerismo y la disposición al diálogo; mientras que en menor grado, se menciona la contribución del teatro foro al mejoramiento de la capacidad de enseñar a ser mejores personas. Los estudiantes también hicieron énfasis en sus necesidades de exploración artística, en la posibilidad que brinda el teatro foro de analizar proposiciones de conflicto emergentes en los ejercicios teatrales de reconstruir la historia, las vivencias cotidianas y de generar alternativas a soluciones de conflictos o problemáticas reales.

Finalmente, y de acuerdo con lo expuesto, los instrumentos aplicados arrojan las siguientes categorías transversales: necesidad de expresarse a través del arte, insuficiencia de estrategias para la gestión de conflictos, nula identificación de los conflictos presentes, el arte y la educación como aprendizajes repetitivos y mecánicos, timidez y temor al interactuar con otras personas, temor a explorar el cuerpo, inseguridad para hablar en público, concepción del teatro como ficción de la realidad, vivencias idealizadas, el teatro solo como entretenimiento, prejuicios sociales, inhibición al expresar situaciones emotivas o vivenciales, insatisfacción por lo que hacen, limitación de la expresión y el pensamiento, e indiferencia a conflictos o problemáticas sociales. 


\section{Conclusiones}

Las conclusiones aquí presentadas aportan a las reflexiones en torno a la gestión de conflictos y la transformación social que presentan los jóvenes universitarios.

Por consiguiente, se concluye que la propuesta de teatro foro como estrategia de transformación social es el principal resultado del presente estudio, responde al objetivo central del trabajo. Parte de la fundamentación teórica, se sustenta de los ejercicios teatrales desarrollados preliminarmente y se estructura en dos fases metodologías: la formulación y la aplicación. En la primera fase, se establece un aprestamiento para la definición de la población objetivo y las herramientas de recolección de información, la aplicación de herramientas, la evaluación del proceso y finalmente, la socialización de los resultados obtenidos; por otra parte, en la segunda fase propuesta, se encamina específicamente al desarrollo del trabajo con el grupo focal, la realización de las entrevistas, la organización y ejecución de los laboratorios de creación basados en el teatro foro y el diligenciamiento del diario de campo; la sistematización y análisis de resultados parciales y la triangulación de las técnicas de recolección de información; la identificación de las conclusiones del trabajo y la socialización y publicación de resultados.

Por lo anterior, en el aprestamiento obtenido se determina que, el proceso se centró en la percepción de cómo los estudiantes estructuran una perspectiva de cada problema planteado y sus necesidades particulares, a través de las interacciones observadas, desarrolladas y sistematizadas en las técnicas de recolección de cada instrumento, donde se identifican experiencias de problemáticas cotidianas y sociales vividas, de esta manera, se evidencian las experiencias, realidades, emociones, sucesos y temas asociados a los conflictos personales de opresión, libremente planteados por cada estudiante desde sus propias percepciones. En este sentido, los jóvenes representaron de manera espontánea las experiencias de opresión vividas en diferentes contextos: conflicto intrafamiliar, violencia contra la mujer, violencia de género, discriminación racial o étnica, matoneo o bulling, conflicto armado, desplazamiento; asimismo, estas narrativas personales e intimas se sistematizaron en las diferentes técnicas de recolección. Evidenciando, que, en los jóvenes universitarios, existe una necesidad de expresarse a través del arte, pero existe temor al explorar el cuerpo, timidez al interactuar con otras personas, inseguridad para hablar en público; asimismo, se reflexiona sobre los aprendizajes mecánicos en el teatro, y su cambio de paradigma de ver el teatro como ficción de la realidad a un teatro que los prepara para asumir esa realidad.

Finalmente, las propuestas artísticas por parte de los jóvenes universitarios, guardan estrecha conexión con las posibles alternativas de solución y aplicación en situaciones cotidianas y reales de conflicto, se evidencia un 
propósito general de mejorar el potencial de aprendizaje y sus relaciones con una proyección social hacia la gestión de conflictos. Se establece que además de las opresiones sociales que los limita en la expresión y el pensamiento, persiste en nuestra sociedad indiferencia a los conflictos o problemáticas sociales y faltan implementar estrategias para la gestión de conflictos. También, es importante señalar que la moderación de las sesiones condujo de manera eficiente para que el grupo lograra los resultados esperados; de este modo, se abordó el manejo de las emociones cuando surgieron y se obtuvieron significados de los estudiantes en su propio lenguaje, además se logró alcanzar un alto nivel de profundización, así como se estimuló la participación y la interacción de todos, procurando que se respetarán los tiempos de intervención y la libertad para expresarse.

\section{REFERENCIAS BIBLIOGRÁFICAS}

Boal, A. (2015). Teatro del oprimido - Teoría y práctica. Buenos Aires, Argentina: Interzona Editora. 17p.

Boal, A. (2012) La estética del oprimido. Traducción de Joana Castells. ISBN: 9788484287414. Barcelona, España: Alba Editorial, 360p.

Boal, A. (2004). El arco iris del deseo. Del teatro experimental a la terapia. España: Alba Ed.

Boal, A. (2001). Teatro del oprimido - Juego para actores y no actores. Barcelona, España: Alba Editorial. 422 p.

Boal, A. (1989). Teatro del oprimido 1. Teoría y práctica, Traducción de Graciela Schmilchuk. ISBN: 968- 429-183-3. México: Nueva imagen, 253 p.

Carr, W. \& Kemmis S. (1986). Teoría crítica de la enseñanza. La investigaciónacción en la formación del profesorado. Martínez Roca, Barcelona. Cap. 5 pp. $140-166$.

Creswell, J.W. (1998). Qualitative inquiry and research design. Choosing among five traditions. Londres: Sage.

De Armas, M. y Carreiro, J. (2013). La mediación en la resolución de conflictos. Barcelona: Editorial: Universidad de las Palmas. 160 pág.

Delors, J. (1996.): "Los cuatro pilares de la educación" en La educación encierra un tesoro. Recuperado de http://uom.uib.cat/digitalAssets/221/221918_9.pdf

Freire, P. (2009). Pedagogía de la esperanza. México. 226 pág.

Freire, P. (1996). Pedagogía de la autonomía. Saberes necesarios para la práctica educativa. México: Siglo XXI editores. 69 pág. 
Freire, P. (1986). Pedagogía del oprimido. Montevideo- Uruguay: Trigésima cuarta edición. Siglo XXI Editores. 245 pág.

Freire, P. (1984). La importancia de leer y el proceso de liberación. Coyoacán, México: Siglo XXI editores. 180 pág.

Freire, P. (1971). La educación como práctica de la libertad. Montevideo - Uruguay: $3^{a}$ edición. Edit. Tierra Nueva. 1pág.

Galtung, J. (2004). Trascender y transformar: Una introducción al trabajo de conflictos. Edit. UNAM-Quimera, México, 2004.

Galtung, J. (2003). Paz por medios pacíficos: Paz y conflicto, desarrollo y civilización. Bilbao, Bakeaz/Gernika Gogoratuz. 354 pág.

Galtung, J. (1981) Hacia una definición de la investigación sobre la paz en UNESCO, Investigación sobre la paz. Tendencias Recientes y Repertorio Mundial, UNESCO, París. P págs. 7-18

Galtung, J. (1976). "Three Approaches to Peace: Peacekeeping, Peacemaking and Peacebuilding." Peace, War and Defense: Essays in Peace Research, Vol. II, Copenhagen: Chritian Ejlers, Pág. 297- 298.

Hernández Arteaga, I., Luna Hernández, J.A. y Cadena Chala, M.C. (2017) Cultura de paz, una construcción desde la educación. Rev. His.edu.latinoam, Vol.19 (28), ISSN: 0122-7238, p. 149-172. DOI: hhtp://doi.org/10.19053/01227238.5596 Recuperado de http://www.scielo.org.co/pdf/rhel/v19n28/v19n28a09.pdf

Lederach, J. (2003) El pequeño libro de la transformación del conflicto. Buenos Libros. Pág. 20.

Lederach, J. (2000). El abecé de la paz y los conflictos. Madrid: La Catarata. 192pág.

Muñoz, B. (2003). "El juego de rol: recurso metodológico para la resolución de conflictos escolares", en Vinyamata, E. (ed.), Aprender del conflicto. Conflictología y Educación, Barcelona: Graó.

Muñoz, F. y Molina, B (2009). Una cultura de paz compleja y conflictiva. La búsqueda de equilibrios dinámicos. Revista de Paz y Conflictos, Núm. 3. Recuperado de http://www.ugr.es/ revpaz/articulos/rpc n3 2010 art3.pdf

Unesco (1998). Resolución A/52/13 de Naciones Unidas, Ginebra.

París, A. y Martínez, H. (2009). El papel de la sociedad civil en la transformación pacífica de conflictos. La búsqueda de equilibrios dinámicos. Castellón, España: Universitat Jaume. 48pág.

Robles, Darwin. J, \& Ortiz. G, Dorys. N. (2017). Educación como práctica social: la cuestión del otro y su reconocimiento. Sophia, Colección de Filosofía de la Educación, (23), 195-218. https://dx.doi.org/10.17163/soph.n23.2017.07 
Robles, Darwin. J, \& Ortiz. G, Dorys. N. (1998). La cultura de paz y la acción de la Unesco en los Estados miembros. Recuperado de http://unesdoc.unesco.org/images/0011/001135/113537s.pdf

Tuvilla, J. (2004). Cultura de paz y educación. Manual de paz y conflictos. Universidad de Granada., 387-426.

Vieites García, M. F. (2015). Augusto Boal en la educación social: del teatro del oprimido al psicodrama silvestre. Foro de Educación, 13(18), págs. 161-179. doi: http://dx.doi.org/10.14516/fde.2015.013.018.009

Vinyamata, E. (2015) Conflictología. Revista de Paz y Conflictos, ISSN 1988-7221 8 (1), Universidad Oberta de Catalunya, pp. 9-24. Recuperado de file:///C:/Users/ASUS/Downloads/Dialnet-Conflictologia-5117130.pdf 